\title{
CHROMOSOMAL MUTAGENESIS IN HUMAN SOMATIC CELLS: 30-YEAR CYTOGENETIC MONITORING AFTER CHORNOBYL ACCIDENT
}

\author{
M.A. Pilinska*, G.M.Shemetun, O.V. Shemetun, S.S. Dybsky, O.B. Dybska, O.O. Talan, L.R. Pedan, D.A. Kurinnyi \\ State Institution "National Research Center for Radiation Medicine \\ of the National Academy of Medical Sciences of Ukraine”, Kyiv 04050, Ukraine
}

\begin{abstract}
In the lecture we have generalized and analyzed the data of cytogenetic laboratory of National Research Center for Radiation Medicine of the National Academy of Medical Sciences of Ukraine on 30-year selective cytogenetic monitoring among the priority contingents of different ages exposed to radiation after Chornobyl accident in Ukraine. It is highlighted that not only targeted but also untargeted radiation-induced cytogenetic effects should be explored, especially in delayed terms following radiation exposure. The new methodical approaches for studying "bystander effect", individual radiosensitivity, and various forms of radiation-induced chromosomal instability (delayed, hidden, transmissible) have been proposed. These approaches proved to be advantageous for analyzing cytogenetic patterns of induction and persistence of chromosomal instability in human somatic cells because of "bystander effect" and "bystander type effect". The phenomenon of positive "reverse" bystander effect has been found. The possibility of modifying the inherited individual human susceptibility to mutagenic exposure by ionizing radiation has been estimated. Finally, the association between hypersensitivity to radiation exposure and realization of oncopathology in exposed individuals has been revealed. The increased intensity of human somatic chromosomal mutagenesis was confirmed not only in the nearest but in the delayed terms following Chornobyl accident as a result of radiation-induced both targeted and untargeted cytogenetic effects. Such effects can be considered as risk factors for malignant transformation of cells, hereditary diseases, birth defects, and multifactorial somatic pathology. This article is a part of a Special Issue entitled "The Chornobyl Nuclear Accident: Thirty Years After".
\end{abstract}

Key Words: Chornobyl accident, targeted and untargeted cytogenetic effects.

As one of the main biological targets for radiation exposure is the genome of somatic and germ cells, exactly the degree of its damage in different terms following irradiation is the criterion of the adverse effects of radiation on human health. Therefore, one of priority scientific directions in radiation genetics considered the study of features the radiation-induced damages in genome structure at different levels of its organization - both direct in directly exposed targeted cells and indirect (untargeted $v s$ disgenomic) - in the unexposed cells.

Among the methods of detection and evaluation the radiation-induced as targeted as well as untargeted genetic effects the leading one still remains cytogenetics - determination of the frequency of chromosome aberrations in human peripheral blood lymphocytes, which in relation to targeted cytogenetic damages was adopted by the International Atomic Energy Agency (IAEA) in 1986 (first edition) and perfected in 2001 (second edition) and 2011 (third edition) as official method of emergency and retrospective biological indication and biological dosimetry of human radiation exposure [1-3]. The third edition was extensively updated to reflect progress made in cytogenetic biological dosimetry during the past decade and cosponsored by the Pan American Health Organization and the World Health Organization.

Cytogenetic examination of exposed persons held since the late 60 -s of the 20 century (when for the first time was developed methods of receiving and analysis

Submitted: August 30, 2016.

*Correspondence: E-mail: pww@ukr.net of human metaphase chromosomes) to nowadays and gradually became an obligatory integral part of medico-radiological control in all cases of emergency human exposure. Since 1986, new contingents of people appeared for cytogenetic survey - persons irradiated as the result of Chornobyl disaster; since 2011 - victims of radiation accident at the nuclear power plant in Fukushima, Japan [4]. In order to improve global readiness of biodosimetric community to large-scale radiation accidents and nuclear terrorism in 2007 under the auspices of the World Health Organization was established international network "BioDoseNet", to which in 2009 was incorporated cytogenetics laboratory of National Research Center for Radiation Medicine of the National Academy of Medical Sciences of Ukraine [5].

During 30 years (1986-2016) scientists from this cytogenetics lab systematically conducted selective cytogenetic monitoring among the critical groups of high priority (around 3500 people of all ages) exposed in Ukraine to Chornobyl accident factors of varying intensity - liquidators with different radiation doses including patients recovered from acute radiation sickness; Chornobyl nuclear power plant and Shelter's staff; evacuees; children and adults from areas contaminated by radionuclides; self-settlers from 30-km alienation zone; some professional groups (foresters, tractor drivers), who lived and worked in areas of heavy radioactive contamination. In the last decade cytogenetic examination of persons who had occupational contact with ionizing radiation while participated in conversion of the "Shelter" into ecologically safe system was carried out $[6,7]$. 
Research of the first twenty years has been mainly focused on the cytogenetic expertise (biological indication and/or biological dosimetry) of human irradiation based on classical targeted cytogenetic effects - the frequency of unstable and stable aberrations of chromosome type that are specific markers of human radiation exposure. Aberrations of chromatid type considered as indicators of radiation-induced chromosome instability. Chromosome aberrations identified as a rule using traditional conventional analysis of uniformly stained metaphase chromosomes and selectively - using G-banding analysis of differentially stained chromosomes and FISH-WCP technology [8-10].

Summarized results of such research, obtained through the first $\sim 15-20$ years after Chornobyl accident allowed to reveal certain patterns of targeted cytogenetic effects in persons of Chornobyl contingent.

In all the observed groups for about two decades remained elevated level of cytogenetic radiation markers.

Mean-group frequencies of targeted radiogenic cytogenetic effects differed depending on the nature, intensity and duration of radiation exposure, time that passed after irradiation; significantly varying individual cytogenetic parameters under identical radiation exposure depend on age and individual radiosensitivity.

The frequency of unstable chromosome aberrations gradually reduced over time (but with preservation of its residual quantity that exceeded spontaneous level for about 20 years), while stable radiogenic markers remained almost at the same level after acute radiation exposure and accumulated under chronic irradiation.

Cytogenetic effect is not always positively correlated with official doses of radiation, which could be associated both with the individual peculiarities of elimination unstable and some part of stable chromosome aberrations as well as with incorrectness of official doses, which was confirmed by us using method FISH-WCP.

In all exposed groups the increase of individual and mean-group frequencies of chromatid type aberrations was observed that could be manifested as radiation-induced chromosome instability (delayed or hidden) that increases the sensitivity of cells to mutagenic exposure of other factors as bystander effect.

Thus, the results of selective cytogenetic monitoring testified destabilization of human genome induced by Chornobyl accident factors, leading among them is ionizing radiation. Dynamics of radiation-induced somatic chromosome mutagenesis was characterized by gradual change not only in the frequency but also the spectra of chromosomal abnormalities that allowed assume the transition from targeted cytogenetic effects to untargeted over time [11].

In the search study of chromosome sensitivity in peripheral blood lymphocytes from exposed persons to testing mutagenic exposure (radiation and chemical) in vitro, we confirmed the reality of formation two oppositely-directed cytogenetic effects - adaptive response in children living in contaminated areas and reduction of chromosome resistance to mutagens in convalescents of acute radiation sickness. Therefore in the remote terms (after $\sim 20$ years) following Chornobyl accident arose the need to conduct fundamental investigations for assessment the radiogenic damage of human genome - identifying not only residual mutagenic effects of ionizing radiation in directly exposed targeted cells, as untargeted effects. To these effects belong various forms of radiation-induced genomic instability - a phenomenon in which the irradiated cells accumulate over time multiple changes that promote the transition of stable genome from normal cells into unstable genome typical in particular for tumor cells, and also bystander effect - secondary changes in the genome of non-irradiated cells [11, 12].

At the cytogenetic level genome instability can be transmitted in the form of potential chromosome damages through several cell generations and realized with delay in vivo or in vitro in the remote terms after radiation exposure (delayed or remote chromosome instability). Part of nonreparated or wrongly reparated chromosome damages in germ cells of irradiated parents can be transmitted into somatic cells of their progeny in the form of various types of chromosome aberrations (transmissible chromosome instability). Hidden chromosome instability manifests as spontaneous or induced hypersensitivity of chromosomes in somatic cells to various mutagens in vivo and in vitro.

Suggest that exactly these long-term effects can play a leading role in the realization as stochastic (especially cancer) as well as some forms of nonstochastic (multifactorial) diseases that caused priority of research in this area within several international projects. In particular, under the auspices of the European Commission and Euratom in 2006-2010 was implemented the Integrated Project NOTE (Nontargeted effects of ionizing radiation). The main objectives of the project were to study the mechanisms of such untargeted effects as radiation-induced genomic instability, radiation-induced bystander effect and adaptive response; study the role of individual radiosensitivity in the modification of untargeted effects; evaluation of possible contribution of untargeted effects into the implementation of health disorders [13].

In our lab purposeful research of radiation-induced untargeted cytogenetic effects was conducted in 2005-2015 in the following main areas [14-17].

Development and introduction of our own experimental models for identification of some untargeted cytogenetic effects:

- for delayed chromosome instability as well as for assessment the persistence of radiation-induced cytogenetic effects - two-termed cultivation of human peripheral blood lymphocytes - not only standard short-term 48-hour incubation, but rarely used long-term cultivation (during 96-144 hours) that allowed to analyze the cells throughout several successive generations; 
- for transmissible chromosome instability - cytogenetic survey of children born to radiationexposed parents with use long-term cultures of lymphocytes;

- for hidden chromosome instability - usage the modified test of provocative mutagenesis (" $\mathrm{G}_{2}$ bleomycin sensitivity assay");

- for bystander effect and bystander type effect - own original model - mixed culture of human peripheral blood lymphocytes that consists from cells exposed in vitro or in vivo (as a source of harmful signal), and non-irradiated cells derived from individuals of the opposite sex, which is used as "bystander"; cytogenetic markers to distinguish between irradiated and non-irradiated populations of lymphocytes are sex chromosomes (Y, XX) and some morphological variants of autosomes determined by G-banding technique.

Study the modifying effects of ionizing radiation on various form of genetically caused chromosome instability under cytogenetic examination of exposed individuals:

- in models in vitro (in successive mitoses);

- in vivo (under cytogenetic examination of exposed persons in different terms following irradiation).

Research the persistence of radiation-induced nontargeted cytogenetic effects over time:

- in models in vitro (in successive mitoses);

- in vivo (under cytogenetic examination of exposed persons in different terms following irradiation).

Research of individual radiosensitivity and features the induction of bystander effect in the age aspect.

The results obtained allowed to establish certain regularities regarding induction and persistence of radiation-induced untargeted cytogenetic effects in human somatic cells at different terms after irradiation in low and high doses, in vitro and in vivo, and make the following general conclusions [18, 19].

The possibility of expression and persistence delayed and probably transmissible chromosome instability in progeny of irradiated parents has been shown that confirmed the high sensitivity of child's organism to mutagenic exposure of ionizing radiation.

The possibility of modification the genetically caused chromosome sensitivity of human somatic cells to mutagenic exposure in vitro as a result of human irradiation in vivo depending on the intensity and nature of radiation exposure was found.

Not only persistence but in some cases increase of chromosomal sensitivity in successive generations of human somatic cells to testing mutagenic exposure in vitro was revealed that leading to higher number hypersensitive persons with hidden chromosomal instability.

The existence of an association between radiationmodified increase of genetically determined individual susceptibility to the testing mutagenic exposure in vitro and the realization of oncopathology (lung cancer) in hypersensitive individuals was determined.
The reality of induction and persistence the dosedependent bystander effect in non-irradiated human peripheral blood lymphocytes, which were cocultured with lymphocytes irradiated in vitro (bystander effect) and in vivo (bystander-type effect) was established. The "reverse" bystander effect - positive impact of non-irradiated cells on irradiated ones during their joint long-term cultivation. The contribution of oxidative stress in the mechanism of radiation-induced bystander effect was confirmed. Significant interindividual variability regarding the degree of chromosome damages in each studied untargeted cytogenetic effects and lack of positive correlation between background and induced frequencies of chromosomal aberrations was established. Significant differences between the spectra of chromosome aberrations when compared targeted and untargeted cytogenetic effects was found, that confirm the correctness of using classical cytogenetic markers of human radiation exposure for retrospective biological dosimetry. Suggest that between main radiation-induced untargeted cytogenetic effects - chromosome instability and bystander effect - exist similarities and interrelation on the mechanism of induction, manifestation and possible contribution to the realization of delayed health effects of human radiation exposure that require further research [13]. Thus, the data have shown the increase of the intensity of somatic chromosome mutagenesis in human not only in the nearest but in the delayed term following the Chornobyl accident as a result of radiation induced both targeted and untargeted cytogenetic effects, confirming the need to continue fundamental cytogenetic studies.

Considering the potential possibility for radiological emergency situations, it is necessary also to intensify the search for new effective and safe radioprotectors capable to prevent or reduce radiation-induced genomic damages in human cells [20]. In 2015 we have begun the studies of the radioprotective properties of astaxanthin (carotenoid from xanthophyll group) not only for cytogenetic, but also epigenetic parameters. Initial results indicate the prospects of astaxanthin as a basis for a future effective radioprotective complex [21].

As in recent few years none of Ukrainian cytogenetic laboratories that has budget funding does not receive reagents and others materials, necessary for such studies, the reality of the implementation the emergency or expert cytogenetic dosimetry is problematic, although the need for such studies periodically occurred. In our opinion, Ukraine should have a special operating lab of "rapid response with the alarming suitcase" for cases of accidental human radiation exposure, which should be in constant readiness for cytogenetic testing.

\section{REFERENCES}

1. Biological Dosimetry: Chromosomal Aberration Analysis for dose Assessment. Technical Report Series. Vienna: International Atomic Energy Agency, 1986. No 260. 
2. Cytogenetic Analysis for Radiation Dose Assessment. A Manual. IAEA. Technical Report Series. Vienna: International Atomic Energy Agency, 2001. No 405.

3. Cytogenetic Dosimetry: Applications in Preparedness for and Response to Radiation Emergencies. Vienna: International Atomic Energy Agency, 2011. 229 p.

4. Imanaka T, Hayashi G, Endo S. Comparison of the accident process, radioactivity release and ground contamination between Chernobyl and Fukushima-1. J Radiat Res 2015; 56 (Suppl): i56-i61.

5. Wilkins RC, Carr Z, Lloyd DC. An update of the WHO Biodosenet: developments since its inception. Radiat Prot Dosimetry 2016 [Epub ahead of print]. DOI: 10.1093/rpd/ncw154.

6. Pilinskaya MA. The results of selective cytogenetic monitoring of Chernobyl accident victims in the Ukraine. Health Phys 1996; 71: 29-33.

7. Pilinskaia MA, Dybskiy SS, Shemetun EV, Dybskaia EB. Somatic chromosome mutagenesis in residents of Ukraine exposed to ionizing radiation in different periods after the Chernobyl accident. Vestn Ross Akad Med Nauk 2011; (9): 63-8 (in Russian).

8. Shemetun OV, Pilinska MA. The detection of stable and unstable markers of radiation action in persons with a history of chronic irradiation using the methods of routine and differentiated staining of metaphase chromosomes. Tsitol Genet 1998; 32: 32-7 (in Ukrainian).

9. Pilinskaia MA, Dybskiy SS, Khaliavka IG. The use of the FISH method for the cytogenetic examination of persons with a history of acute radiation sickness in connection with the accident at the Chernobyl Atomic Electric Power Station. Tsitol Genet 1998; 32: 22-32 (in Russian).

10. Pilinskaia MA, Dybskiy SS, Skaletskiy IuN, et al. The experience of FISH technique application for reconstruction of individual radiation doses in Chernobyl liquidators in the framework of Ukrainian-American project «Leukemia». Tsitol Genet 2006; 40: 34-9 (in Russian).

11. Wright EG. Manifestations and mechanisms of nontargeted effects of ionizing radiation. Mutat Res 2010; 687: $28-33$
12. Shemetun OV, Pilinska MA. Radiation induced bystander effect. Tsitol Genet 2007; 41 (4): 66-71 (in Ukrainian).

13. Kadhim M, Salomaa S, Wright E, et al. Non-targeted effects of ionising radiation - implications for low dose risk. Mutat Res 2013; 752: 84-98.

14. Pilinska MA, Dybsky SS, Dybska OB, Pedan LR. Chromosome instability revealed in children of fathers irradiated during Chernobyl accident. Tsitol Genet 2005; 39: 3240 (in Ukrainian).

15. Pilinska MA, Dybsky SS, Dybska EB, Pedan LR. Radiation-induced modification of the chromosome sensitivity of human somatic cells chromosome sensitivity in testing mutagenic effect of bleomycin. Tsitol Genet 2010; 44: 5864 (in Ukrainian).

16. Pilinskaia MA, Dybskiy SS, Dybskaia EB, Shvaiko LI. Radiation-induced modification of human somatic cells chromosome sensitivity to the testing mutagenic exposure of bleomycin in vitro in lung cancer patients in delayed terms following Chernobyl accident. Tsitol Genet 2012; 46: 36-43 (in Russian).

17. Talan OO, Shemetun OV, Pilinskaya MA. The frequency of spontaneous and radiation-induced cytogenetic damages in persons aged 12-100 years. Eur J Hum Gen 2015; 23 (Suppl): 452.

18. Pilinska MA, Dybsky SS, Dybska OB, et al. Peculiarities of induction and persistence of hidden chromosome instability in peripheral blood lymphocytes of persons occupationally exposed with ionizing radiation. Probl Radiac Med Radiobiol 2014; (19): 321-33.

19. Shemetun EV, Talan OA, Pilinskaia MA. Cytogenetic peculiarities of induction and persistence the bistander effect in human blood lymphocytes. Tsitol Genet 2014; 48: 41-8 (in Russian).

20. Domina EA. Anty-radiation means: classification and mechanisms. Probl Radiac Med Radiobiol 2015; (20): 42-54.

21. Pilinska MA, Kurinnyi DA, Rushkovsky SR, Dybska OB. The impact of astaxanthin on radiation-induced chromosome aberrations in human peripheral blood lymphocytes in vitro. Visn Ukr Soc Genet Select 2016; 14: 52-7 (in Ukrainian). 